Qinglan Xia

\title{
Intersection homology theory via rectifiable currents
}

Received: 21 April 2001 / Accepted: 15 April 2003 /

Published online: 1 July 2003 - (C) Springer-Verlag 2003

\begin{abstract}
Here is given a rectifiable currents' version of intersection homology theory on stratified subanalytic pseudomanifolds. This new version enables one to study some variational problems on stratified subanalytic pseudomanifolds. We first achieve an isomorphism between this rectifiable currents' version and the version using subanalytic chains. Then we define a suitably modified mass on the complex of rectifiable currents to ensure that each sequence of subanalytic chains with bounded modified mass has a convegent subsequence and the limit rectifiable current still satisfies the perversity condition of the approximating chains. The associated mass minimizers turn out to be almost minimal currents and this fact leads to some regularity results.
\end{abstract}

\section{Introduction}

The goal of this paper is to develop a setting for treating variational problems on stratified pseudomanifolds with singularities, such as complex projective varieties. Rather than using the ordinary homology theory on the base space, we will instead use a generalized "homology theory" - the intersection homology theory, introduced by MacPherson and Goresky in the early 1980's ([GM1, GM2]). Such a theory turns out to be more suitable than ordinary homology theory for pseudomanifolds with singularities (see [Kirwan] or [Borel] for details).

In variational problems, one needs to take various limits of e.g. minimizing sequences, but a basic problem is that a limit of geometric intersection chains [GM1] may fail to be a geometric chain; and even if it is, it may not satisfy the important perversity conditions of the approximating chains concerning intersection with singular set. This motivates our use of rectifiable currents with a suitably modified mass norm.

In Sect. 1, we present some necessary preliminaries on the categories of intersection homology theory, geometric measure theory, and subanalytic sets and chains.

In Sect. 2, for a compact stratified subanalytic pseudomanifold, we show how to express the intersection homology groups in terms of integer multiplicity rectifiable currents. These are then isomorphic to the usual intersection homology groups defined by geometric or subanalytic chains with the corresponding perversity conditions. The key idea here involves a technical modification of the proof of

Q. Xia: Department of Mathematics, Rice University, Houston, TX 77005, USA 
the Federer-Fleming's Deformation Theorem [Simon, Sect. 29] to accommodate the perversity condition of intersection homology theory. We study properties of a "safety function" used to quantify the perversity condition for each simplex of the singular locus.

In Sect. 3, we give a suitably modified mass on rectifiable currents such that all rectifiable currents with finite mass and finite boundary mass satisfy automatically the given perversity conditions. Also, by using the Lojasiewicz' inequality of subanalytic sets, we're able to show that all allowable subanalytic chains have finite (modified) mass and finite boundary mass. This fact ensures that our category of rectifiable currents with finite modified mass is still rich enough to contain all the "nice" chains one may consider. Moreover, this modified mass satisfies an important theorem - an analogue of the compactness theorem of Geometric Measure theory (see [F1,Simon]), which implies that each sequence of rectifiable currents with bounded modified mass and boundary mass will have a convergent subsequence and that the limit is a rectifiable current satisfying the perversity conditions of the approximating chains. This property of rectifiable currents overcomes the weakness of geometric chains stated above in the basic problem. The support of the currents we consider may intersect (in a controlled fashion) the singular locus of the pseudomanifold. A related problem with currents avoiding the vertex of a regular cone was studied by [Li].

In Sect. 4, we first achieve an existence theorem for modified mass minimizers. Moreover, we show that these mass minimizers are in fact almost minimizing currents [Bomb]. Thus, by a lemma of Almgren, we achieve a partial regularity theorem for these suitable mass minimizers.

We will, for notational convenience and clarity, restrict to manifolds and pseudomanifolds in $\mathbb{R}^{N}$, although most of our results carry over to more general contexts.

Acknowledgement. The author wants to thank his thesis advisor Robert Hardt for his uncountable help, fruitful discussions and endless encouragement.

\section{Backgrounds}

\subsection{Intersection homology groups $I H^{\bar{p}}(X)$}

As in [Borel, Sect. 1.1], let $X$ be a topological stratified pseudomanifold of dimension $n$ with singular locus $\Sigma$ and a given stratification

$$
X=X_{n} \supset X_{n-2}(=\Sigma) \supset X_{n-3} \supset \cdots \supset X_{0} .
$$

For a triangulation $T$ of $X$, compatible with the stratification, let $C_{*}^{T}(X)$ be the complex of simplicial chains of $T$. Then an element $\xi \in C_{i}^{T}(X)$ is a linear combination

$$
\xi=\sum_{\sigma \in T^{(i)}} \xi_{\sigma} \sigma, \xi_{\sigma} \in \mathbb{Z} .
$$

For $\xi \in C_{i}^{T}(X)$, define $|\xi|$ (the support of $\xi$ ) to be the union of the closures of those i-simplices $\sigma$ for which the coefficient of $\sigma$ in $\xi$ is non-zero. The complex 
$C_{i}(X)$ of all geometric chains of $X$ with integer coefficients is the direct limit of $C_{*}^{T}(X)$ under refinement over all such triangulations of $X$.

Let $\bar{p}=\left(p_{2}, p_{3}, \cdots, p_{n}\right)$, called the perversity, be any fixed nonnegative integers satisfying $p_{2}=0$, and $p_{k} \leq p_{k+1} \leq p_{k}+1$ for all $2 \leq k \leq n$. A geometric chain $\xi$ is said to be $(\bar{p}, i)$ allowable if

$$
\operatorname{dim}_{\mathbb{R}}\left(|\xi| \cap X_{n-k}\right) \leq i-k+p_{k}, \text { for all } k \geq 2 .
$$

The group $I C_{i}^{\bar{p}}(X)$ of intersection chains of dimension $i$ and perversity $\bar{p}$ is the subgroup of geometric chains $\xi \in C_{i}(X)$ such that $\xi$ is $(\bar{p}, i)$ allowable and $\partial \xi$ is $(\bar{p}, i-1)$ allowable.

Definition 1.1.1 The intersection homology groups $I H^{\bar{p}}(X)$ are defined to be the homology groups of the chain complex $I C^{\bar{p}}(X)$.

This definition is independent of the choice of the stratification. See [GM2]

\subsection{Geometric measure theory}

Let $U \subset \mathbb{R}^{N}$ be an open subset and $\mathcal{D}^{i}(U)$ be the set of all differential $i$-forms in $U$ with compact support.

An $i$ dimensional current $T$ on $U$ is a continuous linear functional on $\mathcal{D}^{i}(U)$. Let $\mathcal{D}_{i}(U)$ denote the set of all $i$ dimensional currents on $U$ (see [F1] or [Simon] for more details).

Given a sequence $\left\{T_{j}\right\} \in \mathcal{D}_{i}(U)$, we write $T_{j} \rightarrow T$ in $U$ if $\left\{T_{j}\right\}$ converges weakly to $T \in \mathcal{D}_{i}(U)$ in the usual sense of distributions:

$$
T_{j} \rightarrow T \Longleftrightarrow \lim T_{j}(\omega)=T(\omega), \forall \omega \in \mathcal{D}^{i}(U) .
$$

Given $T \in \mathcal{D}_{i}(U)$, the support of $T$ is defined by

$$
\operatorname{spt} T=U \backslash \cup\{V \subset U \text { open }: \operatorname{spt}(\omega) \subset V \Rightarrow T(\omega)=0\} .
$$

The mass function on $\mathcal{D}_{i}(U)$ is defined by

$$
M(T)=\sup _{\|\omega\| \leq 1, \omega \in \mathcal{D}^{i}(U)} T(\omega) .
$$

More generally, for any open $W \subset U$, we define

$$
M_{W}(T)=\sup _{\|\omega\| \leq 1, \omega \in \mathcal{D}^{i}(U), \operatorname{spt} \omega \subset W} T(\omega) .
$$

Clearly, $M(T)$ is lower semicontinuous under the weak convergence of currents.

An integer multiplicity rectifiable current $T$ is a current coming from an oriented rectifiable set with integer multiplicities (see [F1] or [Simon]). Let $\mathcal{R}_{i}\left(\mathbb{R}^{N}\right)$ be the set of all $i$ dimensional integer multiplicity rectifiable currents in $\mathbb{R}^{N}$ and for any subset $X \subset \mathbb{R}^{N}$, let

$$
\mathcal{R}_{i}(X)=\left\{T \in \mathcal{R}_{i}\left(\mathbb{R}^{N}\right) \mid \operatorname{spt}(T) \subset X\right\} .
$$




\subsection{Subanalytic sets and chains}

According to [Hironaka, proposition 6.11], a subset $A$ of a real-analytic space $\mathrm{X}$ is subanalytic if at any point $a \in A$, there exists an open neighborhood $U$ of $a$ in $X$, a real analytic manifold $Y$ and a finite system of proper real-analytic maps $g_{i j}: Y \rightarrow U, 1 \leq i \leq p$ and $j=1,2$, such that $A \cap U=\cup_{i=1}^{p}\left(\operatorname{Im}\left(g_{i 1}\right)-\operatorname{Im}\left(g_{i 2}\right)\right)$.

Examples of subanalytic subsets of $\mathbb{R}^{N}$ :

(1)analytic varieties;

(2)polyhedrons;

(3)finite unions, intersections, proper projections of subanalytic subsets.

A really important inequality about a subanalytic set is the following (see [Hironaka, 9.5]):

Proposition 1.3.1 (Lojasiewicz' inequality) Let $f$ be a function on $\mathbb{R}^{n}$ with subanalytic graph. Then for each compact subset $K$ of $\mathbb{R}^{n}$, we can find $N \in \mathbb{Z}_{+}$and $C \in \mathbb{R}_{+}$such that for all $x \in K$,

$$
C|f(x)| \geq \operatorname{dist}_{\mathbb{R}^{n}}\left(x, f^{-1}(0)\right)^{N} .
$$

Definition 1.3.2 An integer multiplicity rectifiable current $T \in \mathcal{R}_{i}\left(\mathbb{R}^{N}\right)$ is a subanalytic chain if spt $(T)$ and spt $(\partial T)$ are $i$ and $i-1$ dimensional subanalytic subsets of $\mathbb{R}^{N}$. That is, $T$ is a geometric chain where all the supporting simplices are subanalytic sets.

Definition 1.3.3 A stratified subanalytic pseudomanifold $X$ of dimension $n$ in $\mathbb{R}^{N}$ is a subanalytic pseudomanifold with a stratification

$$
X=X_{n} \supset X_{n-2}(=\Sigma) \supset X_{n-3} \supset \cdots \supset X_{0}
$$

with closed subanalytic subsets $X_{n-k}$ of $\mathbb{R}^{N}$ such that $X_{n-k} \backslash X_{n-k-1}$ is empty or a subanalytic manifold of dimension $n-k$ and such that the local normal triviality holds in the subanalytic category. See [Hardt3] for details.

\subsection{General setup}

For the rest of the paper, we let $X \subset \mathbb{R}^{N}$ be a compact stratified subanalytic pseudomanifold with singular set $\Sigma$ and a given stratification

$$
X=X_{n} \supset X_{n-2}(=\Sigma) \supset X_{n-3} \supset \cdots \supset X_{0} .
$$

Also, let $\bar{p}$ be a fixed perversity function (as in Sect. 1.1). A rectifiable current $T \in \mathcal{R}_{i}(X)$ is said to be $(\bar{p}, i)$ allowable if

$$
\operatorname{dim}\left(\operatorname{spt}(T) \cap X_{n-k}\right) \leq i-k+p_{k}, \text { for all } k \geq 2 .
$$

Finally, we fix an integer $i \in\{0,1, \cdots, n\}$ and consider various dimensional chains in $X$. Let

$$
\mathcal{R}_{i}(X)=\left\{T \in \mathcal{R}_{i}\left(\mathbb{R}^{N}\right) \mid s p t T \subset X\right\}
$$

$\mathcal{P}_{i}(X)=\left\{T \in \mathcal{R}_{i}(X) \mid T\right.$ is $(\bar{p}, i)$ allowable and $\partial T$ is $(\bar{p}, i-1)$ allowable $\} ;$

$\mathcal{S}_{i}(X)=\left\{T \in \mathcal{P}_{i}(X)\right.$ subanalytic chain $\}$. 


\section{Intersection homology theory in rectifiable currents' version}

Here we prove that intersection homology theory defined via rectifiable currents coincides with the usual definition [GM1] involving geometric chains or subanalytic chains.

Lemma 2.0.1 There exists a triangulation $\mathcal{T}$ of $(X, \Sigma)$, compatible with the given stratification, such that:

For all open simplices $\sigma \subset \Sigma$ of $\mathcal{T}$, if $\sigma \subset X_{n-j} \backslash X_{n-j-1}$ for some $2 \leq j \leq n$, then $\partial \sigma \cap X_{n-j-1}$ is either empty or a face of $\partial \sigma$.

Proof. By [Hardt4], there exists a subanalytic triangulation of $(X, \Sigma)$, compatible with the given stratification. Subanalytically subdividing this triangulation, one get a triangulation with the desired properties.

From now on, we fix this triangulation $\mathcal{T}$ and denote

$$
\mathcal{T}_{\Sigma}=\{\text { open simplices } \sigma \in \mathcal{T}: \sigma \subset \Sigma\}
$$

For notational convenience, we rephrase the perversity condition using the following

Definition 2.0.2 The $i$-th safety function $s: \mathcal{T}_{\Sigma} \rightarrow \mathbb{R}$ is given by:

$$
s(\sigma):=i-j+p_{j}-1 \text {, if } \sigma \subset X_{n-j} \backslash X_{n-j-1} .
$$

Remark 2.0.3. Now, $T \in \mathcal{P}_{i}$ if and only if for any $\sigma \in \mathcal{T}_{\Sigma}$,

$$
\operatorname{dim} \operatorname{spt}(T) \cap \sigma \leq s(\sigma)+1 \text { and } \operatorname{dim} \operatorname{spt}(\partial T) \cap \sigma \leq s(\sigma)
$$

Proposition 2.0.4 If $\sigma_{1}$ is a face of $\sigma_{2}$ (denoted by $\left.\sigma_{1} \prec \sigma_{2}\right)$, then $s\left(\sigma_{1}\right) \leq s\left(\sigma_{2}\right)$. This means the interior simplex is "safer" than the boundary.

Proof. One may assume that $\sigma_{1} \subset X_{n-j-1} \backslash X_{n-j-2}$ and $\sigma_{2} \subset X_{n-j} \backslash X_{n-j-1}$ for some $2 \leq j \leq n$. Then

$$
s\left(\sigma_{1}\right)-s\left(\sigma_{2}\right)=i-(j+1)+p_{j+1}-1-\left(i-j+p_{j}-1\right)=p_{j+1}-p_{j}-1 \leq 0 .
$$

Lemma 2.0.5 Suppose $\sigma_{1}, \sigma_{2} \in \mathcal{T}_{\Sigma}$. Let $\tau \in \mathcal{T}_{\Sigma}$ be the open simplex of minimum dimension such that $\sigma_{1} \prec \bar{\tau}, \sigma_{2} \prec \bar{\tau}$. Then

$$
s(\tau)=\max \left(s\left(\sigma_{1}\right), s\left(\sigma_{2}\right)\right) .
$$

Proof. Otherwise, by the Proposition 2.0.4, $s\left(\sigma_{1}\right)<s(\tau)$ and $s\left(\sigma_{2}\right)<s(\tau)$. This implies that if $\tau \subset X_{n-j} \backslash X_{n-j-1}$, then $\bar{\sigma}_{1}, \bar{\sigma}_{2} \subset X_{n-j-1} \cap \partial \tau$. From the property 2.0.1, $\partial \tau \cap X_{n-j-1}$ is a unique closed simplex which is denoted by $\bar{\sigma}$. Therefore, we have $\sigma_{1} \prec \bar{\sigma}$ and $\sigma_{2} \prec \bar{\sigma}$, which contradicts to the minimum dimension property of $\tau$. 
For an open simplex $\sigma \in \mathcal{T}$, let

$$
\text { st }(\sigma)=\cup\{\tau \in \mathcal{T}: \sigma \prec \tau\}
$$

be the open star of $\sigma$ and $S t(\sigma)=\overline{s t(\sigma)}$ denotes the closed star of $\sigma$.

For the rest of this section, we fix one rectifiable current $T \in \mathcal{P}_{i}(X)$. Our goal is to deform $T$ to an allowable subanalytic chain $S \in \mathcal{S}_{i}(X)$ using allowable currents. To achieve this, we make the following technical definitions.

Definition 2.0.6 Given an open simplex $\sigma \in \mathcal{T}_{\Sigma}$.

1. $\sigma$ is absolutely good if $\operatorname{dim} \sigma \leq s(\sigma)$;

2. $\sigma$ is good w.r.t. T of type (I) if $\sigma$ is absolutely good;

$\sigma$ is good w.r.t. T of type (II) if $\operatorname{dim}(\sigma)=s(\sigma)+1$ and $\operatorname{spt}(\partial T) \cap s t(\sigma)=\emptyset$;

$\sigma$ is good w.r.t. $T$ of type (III) if spt $(T) \cap$ st $(\sigma)=\emptyset$.

3. $\sigma$ is bad w.r.t. $T$ if $\sigma$ is not good w.r.t. T, i.e.

$$
\operatorname{dim} \sigma>s(\sigma), \operatorname{spt}(T) \cap s t(\sigma) \neq \emptyset
$$

and also spt $(\partial T) \cap s t(\sigma) \neq \emptyset$ in case $\operatorname{dim}(\sigma)=s(\sigma)+1$.

Note that $\sigma \in \mathcal{T}_{\Sigma}$ being absolutely good trivially gives the perversity condition (1) for any $\tilde{T} \in \mathcal{R}_{i}(X)$.

To make the inductive argument in our deformation theorem 2.0.12, we will first fix any open simplex $\sigma_{0}$ of minimum dimension in the family

$$
\{\tau: s(\tau)=\min \{s(\sigma): \sigma \text { is bad w.r.t. } T\}\} .
$$

Lemma 2.0.7 Any face $\sigma_{1}$ of $\partial \sigma_{0}$ is good w.r.t. T of types I or II.

Proof. By the Proposition 2.0.4, $s\left(\sigma_{1}\right) \leq s\left(\sigma_{0}\right)$. Since $\operatorname{dim} \sigma_{1}<\operatorname{dim} \sigma_{0}$, by the minimum of $\sigma_{0}, \sigma_{1}$ is good w.r.t. $T$. On the other hand, the fact $s t\left(\sigma_{0}\right) \subset \operatorname{st}\left(\sigma_{1}\right)$ implies $\operatorname{spt}(T) \cap s t\left(\sigma_{1}\right) \supseteq \operatorname{spt}(T) \cap s t\left(\sigma_{0}\right) \neq \emptyset$. i.e. $\sigma_{1}$ is not good w.r.t. $T$ of types III. Thus, $\sigma_{1}$ is good w.r.t. $T$ of types either I or II.

Proposition 2.0.8 For $T$ and $\sigma_{0}$ as above, there exists a $T_{1} \in \mathcal{P}_{i}$ and $R \in \mathcal{P}_{i+1}$, $L \in \mathcal{P}_{i}$ such that

(a) $T=T_{1}+\partial R+L$;

(b) $\left\{\sigma: \sigma\right.$ is bad w.r.t. $\left.T_{1}\right\} \varsubsetneqq\{\sigma: \sigma$ is bad w.r.t. $T\}$.

(c) $L=0$ if $\partial T=0$;

(d) $L \in \mathcal{S}_{i}$ if $\partial T \in \mathcal{S}_{i-1}$.

Proof. We'll obtain $T_{1}$ as $p_{\#} T$ for a suitable map $p$ constructed differently in the two possible cases:

Case 1: $\operatorname{dim} \sigma_{0}>s\left(\sigma_{0}\right)+1$.

Since

$$
\operatorname{dim} \sigma_{0}>s\left(\sigma_{0}\right)+1 \geq \operatorname{dim} \operatorname{spt}(T) \cap \bar{\sigma}_{0},
$$


there exists a point $a_{0} \in \sigma_{0} \backslash s p t(T)$. Let

$$
p: S t\left(\sigma_{0}\right) \backslash a_{0} \rightarrow \partial\left(s t\left(\sigma_{0}\right)\right)
$$

be the "radial retraction" of $S t\left(\sigma_{0}\right)$ with $a_{0}$ as origin (see [Simon, p. 166]). Outside the closed star $S t\left(\sigma_{0}\right)$, one may extend it to be the identical map.

Now, let's show that $p_{\#} T \in \mathcal{P}_{i}$. It's sufficient to show that for any $\sigma \in \mathcal{T}_{\Sigma}$ $\cap S t\left(\sigma_{0}\right)$ which is not absolutely good,

$$
\operatorname{dim} s p t\left(p_{\#} T\right) \cap \sigma \leq s(\sigma)+1 \text { and } \operatorname{dim} s p t\left(\partial p_{\#} T\right) \cap \sigma \leq s(\sigma) .
$$

In fact, if $\sigma$ is a face of $\partial \sigma_{0}$, then by the Lemma 2.0.7, $\sigma$ is good w.r.t. $T$ of type III; i.e. $\operatorname{dim} \sigma=s(\sigma)+1$ and $\operatorname{spt}(\partial T) \cap s t(\sigma)=\emptyset$. Thus, $\operatorname{dim} \operatorname{spt}\left(p_{\#} T\right) \cap \sigma \leq$ $\operatorname{dim} \sigma \leq s(\sigma)+1$ and $\operatorname{spt}\left(\partial p_{\#} T\right) \cap \sigma=\operatorname{spt}\left(p_{\#} \partial T\right) \cap \sigma=\operatorname{spt}(\partial T) \cap \sigma=\emptyset$. Therefore, $\sigma$ satisfies the identity (2).

If $\sigma$ belongs to the open star st $\left(\sigma_{0}\right)$, then by the definition of radial retraction, $\sigma \cap \operatorname{spt}\left(p_{\#} T\right)=\emptyset$ and hence $\sigma$ satisfies (2).

If $\sigma$ belongs to $\partial s t\left(\sigma_{0}\right) \backslash \partial \sigma_{0}$, then there are three subcases:

Subcase 1: $\sigma$ is good w.r.t. $T$ of type III . i.e. $s t(\sigma) \cap \operatorname{spt}(T)=\emptyset$. This is a trivial case, because $s t(\sigma) \cap s p t\left(p_{\#} T\right)=\emptyset$ is still true and hence $\sigma$ satisfies (2) and is good w.r.t. $p_{\#} T$ of type II.

Subcase 2: $\sigma$ is good w.r.t. $T$ of type II . i.e. $\operatorname{dim} \sigma=s(\sigma)+1$ and $\operatorname{spt}(\partial T) \cap$ $s t(\sigma)=\emptyset$. This is also a trivial case, because one still has $\operatorname{dim} \sigma=s(\sigma)+1$ and $s t(\sigma) \cap \operatorname{spt}\left(p_{\#} \partial T\right)=\emptyset$. Hence $\sigma$ satisfies (2) and is good w.r.t. $p_{\#} T$ of type II.

Subcase 3: $\sigma$ is bad w.r.t. T. Then, $s(\sigma) \geq s\left(\sigma_{0}\right)$ and we choose $\tau \in \mathcal{T}_{\Sigma}$ to be the open simplex of minimum dimension such that $\sigma_{0} \prec \tau$ and $\sigma \prec \tau$. Since $s\left(\sigma_{0}\right) \leq s(\sigma) \leq s(\tau)$, the Lemma 2.0.5 implies $s(\sigma)=s(\tau)$. Now, note that

$$
\begin{aligned}
& \operatorname{dim} \operatorname{spt}\left(p_{\#} T\right) \cap \sigma \leq \max (\operatorname{dim} \operatorname{spt}(T) \cap \tau, \operatorname{dim} s p t(T) \cap \sigma) \\
& \leq s(\tau)+1=s(\sigma)+1 ; \\
& \operatorname{dim} \operatorname{spt}\left(\partial\left(p_{\#} T\right)\right) \cap \sigma=\operatorname{dim} \operatorname{spt}\left(\left(p_{\#} \partial T\right)\right) \cap \sigma \\
& \quad \leq \max (\operatorname{dim} \operatorname{spt}(\partial T) \cap \tau, \operatorname{dim} \operatorname{spt}(\partial T) \cap \sigma) \leq s(\tau)=s(\sigma) .
\end{aligned}
$$

Therefore, $\sigma$ satisfies (2).

This shows that $p_{\#} T \in \mathcal{P}_{i}$.

Case 2: $\operatorname{dim} \sigma_{0}=s\left(\sigma_{0}\right)+1$. In this case, $\operatorname{spt}(\partial T) \cap s t\left(\sigma_{0}\right) \neq \emptyset$.

Here we will use a different formula for $p$. Since $T \in \mathcal{P}_{i}$, by the identity (1), $\operatorname{dim}\left(\operatorname{spt}(\partial T) \cap \sigma_{0}\right) \leq s\left(\sigma_{0}\right)<\operatorname{dim} \sigma_{0}$. We choose a point $a_{0} \in \sigma_{0} \backslash \operatorname{spt}(\partial T)$ with $\operatorname{dist}\left(a_{0}, \sigma_{0} \backslash \operatorname{spt}(\partial T)\right)>\epsilon$ for some small positive constant $\epsilon$ (It is possible that $\left.a_{0} \in \operatorname{spt}(T)\right)$. Define a help function $f:[0,+\infty) \rightarrow[0,+\infty)$ by

$$
f(t)=\left\{\begin{array}{l}
\frac{t}{\epsilon}, 0 \leq t<\epsilon \\
1, \epsilon \leq t \leq 1 \\
t, t>1
\end{array}\right.
$$


Now, for any $x \in$ st $\left(\sigma_{0}\right)$, let $\gamma_{x}:[0,1] \rightarrow X$ be the "radial arc" starting at $a_{0}$, passing through $x$ and ending at a point $\gamma_{x}(1)$ on $\partial s t\left(\sigma_{0}\right)$. Now we define $p: X \rightarrow X$ by

$$
p(x)= \begin{cases}\gamma_{x}\left(f\left(\frac{\operatorname{dist}(x, a)}{\operatorname{dist}\left(\gamma_{x}(1), a\right)}\right)\right) & , \text { for } x \in S t\left(\sigma_{0}\right) ; \\ x & \text { for } x \in X \backslash S t\left(\sigma_{0}\right) .\end{cases}
$$

To describe this map geometrically, let $U_{\epsilon}$ be the small star neighborhood of the point $a$, similar to $s t\left(\sigma_{0}\right)$ but scaled down by a factor $\epsilon$. Then the map $p$ fixes all points outside $s t\left(\sigma_{0}\right)$, maps $U_{\epsilon}$ homothetically to $s t\left(\sigma_{0}\right)$ and projects the remaining "annular region" radially to $\partial\left(S t \sigma_{0}\right)$. Clearly, this map is Lipschitz (with Lipschitz constant $\frac{C}{\epsilon}$ ). We need to show $p_{\#} T \in \mathcal{P}_{i}$.

As in case 1, it's sufficient to show (2) for any $\sigma \in \mathcal{T}_{\Sigma} \cap S t\left(\sigma_{0}\right)$ which is not absolutely good. We treat all the possible subcases as before:

If $\sigma$ belongs to the open star st $\left(\sigma_{0}\right)$ i.e. $\sigma_{0} \prec \sigma$, then since $\operatorname{spt}(\partial T) \subset X \backslash U_{\epsilon}$, we have $\operatorname{spt}\left(\partial p_{\#} T\right)=\operatorname{spt}\left(p_{\#} \partial T\right) \subset X \backslash s t\left(\sigma_{0}\right)$. Thus, the fact $s t(\sigma) \subset \operatorname{st}\left(\sigma_{0}\right)$ implies $\operatorname{spt}\left(\partial p_{\#} T\right) \cap s t(\sigma)=\emptyset$. On the other hand,

$$
\operatorname{dim} \operatorname{spt}\left(p_{\#} T\right) \cap \sigma=\operatorname{dim} \operatorname{spt}(T) \cap \sigma \leq s(\sigma)+1 .
$$

Therefore, $\sigma$ also satisfies (2).

If $\sigma$ belongs to the boundary $\partial \sigma_{0}$, then by the Lemma 2.0.7, $\sigma$ is good w.r.t. $T$ of type II; i.e. $\operatorname{dim} \sigma=s(\sigma)+1$ and $\operatorname{spt}(\partial T) \cap s t(\sigma)=\emptyset$. Now, $\operatorname{dim} s p t\left(p_{\#} T\right) \cap \sigma \leq$ $\operatorname{dim} \sigma=s(\sigma)+1$ and $\operatorname{spt}\left(\partial p_{\#} T\right) \cap \sigma=\emptyset$. Hence, $\sigma$ satisfies (2).

If $\sigma$ belongs to $\partial s t\left(\sigma_{0}\right) \backslash \partial \sigma_{0}$, then we choose $\tau \in \mathcal{T}_{\Sigma}$ to be the open simplex of minimum dimension such that $\sigma_{0} \prec \tau$ and $\sigma \prec \tau$. Since $s\left(\sigma_{0}\right) \leq s(\sigma) \leq s(\tau)$, the Lemma 2.0.5 implies $s(\sigma)=s(\tau)$. Thus,

$$
\begin{aligned}
\operatorname{dim} \operatorname{spt}\left(p_{\#} T\right) \cap \sigma & \leq \max (\operatorname{dim} \operatorname{spt}(T) \cap \tau, \operatorname{dim} \operatorname{spt}(T) \cap \sigma) \\
& \leq s(\tau)+1=s(\sigma)+1
\end{aligned}
$$

and

$$
\begin{gathered}
\operatorname{dim} \operatorname{spt}\left(\partial p_{\#} T\right) \cap \sigma=\operatorname{dim} \operatorname{spt}\left(p_{\#} \partial T\right) \cap \sigma \\
\leq \max (\operatorname{dim} \operatorname{spt}(\partial T) \cap \tau, \operatorname{dim} \operatorname{spt}(\partial T) \cap \sigma) \leq s(\tau)=s(\sigma) .
\end{gathered}
$$

Therefore, $\sigma$ satisfies (2).

This shows that also in case $2, p_{\#} T \in \mathcal{P}_{i}$.

Now, let $T_{1}=p_{\#} T$ and let $h$ be an "affine homotopy" from the identity to $p$, $R=h_{\sharp}([[0,1]] \times T)$, and $L=h_{\sharp}([[0,1]] \times \partial T)$. Then for any $\sigma \in \mathcal{T}_{\Sigma}$,

$$
\operatorname{dim}(R \cap \bar{\sigma}) \leq \operatorname{dim}(T \cap \bar{\sigma})+1 \leq i-j+p_{j}+1,
$$

and

$$
\operatorname{dim}(\partial R \cap \bar{\sigma}) \leq \operatorname{dim}(\partial T \cap \bar{\sigma})+1 \leq i-j+p_{j},
$$


so $R \in \mathcal{P}_{i+1}$. Also,

$$
\operatorname{dim} L \leq \operatorname{dim}(\partial T \cap \bar{\sigma})+1 \leq(i-1)-j+p_{j}+1=i-j+p_{j},
$$

so $L \in \mathcal{P}_{i}$.

(a) now follows from the homotopy formula [Simon, pg 139].

To prove (b), note that if $\sigma$ is good w.r.t. $T$, then $\sigma$ is still good w.r.t. $p_{\#} T$. Also, there is one open simplex, namely $\sigma_{0}$, which is good w.r.t. $p_{\#} T$ but bad w.r.t. $T$. Thus, we have

$$
\left\{\sigma: \sigma \text { is bad w.r.t. } p_{\#} T\right\} \varsubsetneqq\{\sigma: \sigma \text { is bad w.r.t. } T\} \text {. }
$$

(c) and (d) readily follow from the definition of $L$.

Corollary 2.0.9 For any $T \in \mathcal{P}_{i}$, there exists a $T_{1} \in \mathcal{P}_{i}$ and $R \in \mathcal{P}_{i+1}, L \in \mathcal{P}_{i}$ such that

(a) $T=T_{1}+\partial R+L$;

(b) All open simplices $\sigma \in \mathcal{T}_{\Sigma}$ are good w.r.t. $T_{1}$;

(c) $L=0$ if $\partial T=0$;

(d) $L \in \mathcal{S}_{i}$ if $\partial T \in \mathcal{S}_{i-1}$.

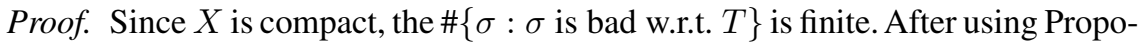
sition 2.0.8, we may apply it a second time with $T$ replaced by $T_{1}$ (and new choice of $\left.\sigma_{0}\right)$. Continuing inductively a finite number of times, one will get the desired results.

Lemma 2.0.10 Suppose $T \in \mathcal{P}_{i}$ with spt $T \subset \mathcal{T}_{k}$, the $k$-skeleton of $\mathcal{T}$, for some $k \geq i+1$. If all open simplices $\sigma \in \mathcal{T}_{\Sigma}$ are good w.r.t. $T$, then there exists $T_{1} \in \mathcal{P}_{i}$ with spt $T_{1} \subset \mathcal{T}_{k-1}, R \in \mathcal{P}_{i+1}$ and $L \in \mathcal{P}_{i}$ such that

(a) all open simplices $\sigma \in \mathcal{T}_{\Sigma}$ are good w.r.t. $T_{1}$,

(b) $T=T_{1}+\partial R+L$

(c) $L=0$ if $\partial T=0$

(d) $L \in \mathcal{S}_{i}$ if $\partial T \in \mathcal{S}_{i-1}$

Proof. As in [Simon, Lemma 29.4], one can choose, for each k-simplex $\tau$ of $\mathcal{T}_{k}$, a suitable point $a_{\tau} \in \tau$ so that the radial retraction away from $a_{\tau}$ gives a locally Lipschitz map $\psi: \mathcal{T}_{k} \backslash \cup_{\tau}\left\{a_{\tau}\right\} \rightarrow \mathcal{T}_{k-1}$ along with a mass bound for $\psi_{\#} T$.

For any $\sigma \in \mathcal{T}_{\Sigma}$, we'll show that $\sigma$ is also good w.r.t. $T_{1}=\psi_{\#} T$. A basic fact about the map $\psi$ is that $\psi^{-1}(x) \subset$ st $(\sigma)$ for any $x \in \sigma$ and hence $\psi^{-1}(s t(\sigma)) \subset$ st $(\sigma)$. Therefore, if $\sigma$ is absolutely good, then $\sigma$ is automatically good w.r.t. $T_{1}$ of type I. If $\sigma$ is good w.r.t. $T$ of type II, i.e. $\operatorname{dim}(\sigma)=s(\sigma)+1$ and $\operatorname{spt}(\partial T) \cap$ $s t(\sigma)=\emptyset$, then $\operatorname{dim} T_{1} \cap \sigma \leq \operatorname{dim} \sigma=s(\sigma)+1$ and $\operatorname{spt}\left(\partial T_{1}\right) \cap \operatorname{st}(\sigma) \subset$ $\psi(\operatorname{spt}(\partial T) \cap s t(\sigma))=\emptyset$. Hence $\sigma$ is good w.r.t. $T_{1}$ of type II. Finally, if $\sigma$ is good w.r.t. $T$ of type III, i.e. $\operatorname{spt}(T) \cap \operatorname{st}(\sigma)=\emptyset$, then $\operatorname{spt}\left(T_{1}\right) \cap \operatorname{st}(\sigma) \subset$ $\psi(\operatorname{spt}(T) \cap s t(\sigma))=\emptyset$. Hence $\sigma$ is also good w.r.t. $T_{1}$ of type III. This shows that all open simplices $\sigma \in \mathcal{T}_{\Sigma}$ are still good w.r.t. $T_{1}$.

Now as usual, let $h$ be an "affine homotopy" from the identity to $\psi, R=$ $h_{\sharp}([[0,1]] \times T)$, and $L=h_{\sharp}([[0,1]] \times \partial T)$. One readily checks that $R \in \mathcal{P}_{i+1}$ and $L \in \mathcal{P}_{i}$ have the desired properties. 
By applying this lemma repeatedly, we will eventually get $\operatorname{spt}(T) \subset \mathcal{T}_{i}$, then we'll use the following:

Lemma 2.0.11 Suppose $T \in \mathcal{P}_{i}$ with spt $T \subset \mathcal{T}_{i}$. If all open simplices $\sigma \in \mathcal{T}_{\Sigma}$ are good w.r.t. $T$, then there exists $S=\Sigma_{F \in \mathcal{T}_{i}} \beta_{F}[[F]] \in \mathcal{S}_{i}$ for some integers $\beta_{F}$ such that $M(T-S)+M(\partial(T-S)) \leq c M(\partial T)$ for some constant $c$.

Proof. As in [Simon, pp. 175-176], for any $i$-dimensional face $F$, one can find an integer $\beta_{F}$ such that $M\left(T\left\llcorner F-\beta_{F}[[F]]\right)+M\left(\partial\left(T\left\llcorner F-\beta_{F}[[F]]\right)\right) \leq\right.\right.$ $c M\left(\partial T\llcorner F)\right.$. Now, we'll show that $S=\Sigma_{F \in \mathcal{T}_{i}} \beta_{F}[[F]] \in \mathcal{P}_{i}$. In fact, for any $\sigma \in \mathcal{T}_{\Sigma}$ we know that $\sigma$ is good w.r.t. $T$. We'll show that $\sigma$ is also good w.r.t. $S$. If $\sigma$ is absolutely good, then $\sigma$ is automatically good w.r.t. $S$. If $\sigma$ is good w.r.t. $T$ of type II, i.e. $\operatorname{dim}(\sigma)=s(\sigma)+1$ and $\operatorname{spt}(\partial T) \cap s t(\sigma)=\emptyset$. This implies that for any $F \in \mathcal{T}_{i} \cap s t(\sigma)$, we have $\partial T\left\llcorner F=0\right.$. Therefore, $T\left\llcorner F=\beta_{F}[[F]]\right.$ and hence $\operatorname{spt}(\partial S) \cap s t(\sigma)=\operatorname{spt}(\partial T) \cap \operatorname{st}(\sigma)=\emptyset$. Thus, $\sigma$ is also good w.r.t. $S$ of type II. Finally, if $\sigma$ is good w.r.t. $T$ of type III, i.e. $\operatorname{spt}(T) \cap \operatorname{st}(\sigma)=\emptyset$, then for any $F \in \mathcal{T}_{i} \cap s t(\sigma), T\left\llcorner F=0\right.$ and hence $\beta_{F}=0$. This also shows $\operatorname{spt}(S) \cap \operatorname{st}(\sigma)=\emptyset$ and $\sigma$ is good w.r.t. $S$ of type III. Therefore, all open simplices $\sigma \in \mathcal{T}_{\Sigma}$ are good w.r.t. $S$, which automatically implies $S \in \mathcal{P}_{i}$. By the construction of $S$, we know that $S$ is a subanalytic chain.

Theorem 2.0.12 (Deformation Theorem) For any $T \in \mathcal{P}_{i}$, there exists a $S \in \mathcal{S}_{i}$ and $R \in \mathcal{P}_{i+1}, L \in \mathcal{P}_{i}$ such that
(a) $T=S+\partial R+L$
(b) $L=0$ if $\partial T=0$
(c) $L \in \mathcal{S}_{i}$ if $\partial T \in \mathcal{S}_{i-1}$

Proof. First apply Corollary 2.0.9 to change $T$ so that all the open simplices are good w.r.t. $T$, then apply Lemma 2.0.10 inductively, we may assume $\operatorname{spt} T \subset \mathcal{T}_{i}$. At last, apply Lemma 2.0.11.

Definition 2.0.13 Let $I H_{*}^{\text {subanalytic }}(X), I H_{*}(X)$ denote the homology groups of the chain complexes $\left\{\mathcal{S}_{i}\right\},\left\{\mathcal{P}_{i}\right\}$ defined above respectively.

Then, we have the following isomorphism theorem:

Theorem 2.0.14 (Isomorphism Theorem) The inclusion map

$j: I H_{*}^{\text {subanalytic }}(X) \hookrightarrow I H_{*}(X)$ is an isomorphism.

Proof. (i) $j$ is injective.

If $[S]=[0] \in I H_{i}(X)$, i.e. $S=\partial T$ for some $T \in \mathcal{P}_{i+1}(X)$, then by the deformation Theorem 2.0.12,

$$
T=S^{\prime}+\partial R^{\prime}+L^{\prime}
$$

with $L^{\prime}$ subanalytic. So, $S=\partial\left(S^{\prime}+L^{\prime}\right)$ and $S^{\prime}+L^{\prime} \in \mathcal{S}_{i+1}$. Hence, $j$ is injective.

(ii) $j$ is onto. For any $[T] \in I H_{i}(X)$, by the deformation Theorem 2.0.12, $T=S+\partial R$. Hence, $[T]=[S]=j([S])$, i.e. $j$ is onto. 


\section{A modified mass on the complex of rectifiable currents}

The limit of a sequence of rectifiable currents with bounded mass and bounded boundary mass is a rectifiable current [Simon, Theorem 27.3]. However, the limit rectifiable current may fail to satisfy the allowability conditions of the approximating chains. This motivates us to modify the usual mass by adding some suitable mass modifiers. For any rectifiable current $T \in \mathcal{R}_{i}(X)$ and each singular stratum, we'll add a mass modifier for $T$ corresponding to that stratum. To control the amount of mass modified, we choose and fix a small tolerance $\delta>0$.

As in Sect. 1.4, we have a fixed perversity $\bar{p}=\left(p_{2}, \cdots, p_{n}\right)$ and a fixed dimension $i \in\{0, \cdots, n\}$. Now, for each singular stratum $X_{n-k}$ with some integer $k \in\{2, \cdots, n\}$ and any rectifiable current $T \in \mathcal{R}_{i}(X)$, we'll define a $k$-th mass modifier $m_{k}^{\delta}(T)$ in the 3 possible cases as follows:

Case 1: $i-k+p_{k} \geq n-k$, i.e. $i+p_{k} \geq n$ (e.g. $i=n$ ).

In this case,

$$
\operatorname{dim}\left(\operatorname{spt}(T) \cap X_{n-k}\right) \leq \operatorname{dim}\left(X_{n-k}\right) \leq n-k \leq i-k+p_{k} .
$$

i.e. the perversity condition is automatically satisfied. Since it's unnecessary to make any modification on the mass, we here set $m_{k}^{\delta}(T):=0$.

Case 2: $i-k+p_{k}<0$, i.e. $i<k-p_{k}$. e.g. $i=0$ or 1 .

In this case, $\operatorname{dim}\left(\operatorname{spt}(T) \cap X_{n-k}\right) \leq i-k+p_{k}$ iff $\operatorname{spt}(T) \cap X_{n-k}=\emptyset$.

Here we define the $k$-mass modifier

$$
m_{k}^{\delta}(T):=\ln \frac{\delta}{\operatorname{dist}\left(\operatorname{spt}(T), X_{n-k}\right)} M\left(T\left\llcorner B\left(X_{n-k}, \delta\right)\right) .\right.
$$

Before considering the remaining case, we first make two easy but important observations:

Lemma 3.0.15 $\operatorname{dim}\left(\operatorname{spt}(T) \cap X_{n-k}\right) \leq i-k+p_{k}$ iff $m_{k}^{\delta}(T)<+\infty$.

Proof.

$$
\begin{gathered}
m_{k}^{\delta}(T)<+\infty \Leftrightarrow \operatorname{dist}\left(\operatorname{spt}(T), X_{n-k}\right)>0 \\
\Leftrightarrow \operatorname{spt}(T) \cap X_{n-k}=\emptyset \Leftrightarrow \operatorname{dim}\left(\operatorname{spt}(T) \cap X_{n-k}\right) \leq i-k+p_{k} .
\end{gathered}
$$

Lemma 3.0.16 $m_{k}^{\delta}$ is lower-semi continuous with respect to the weak convergence of currents.

Proof. If $T_{j} \rightarrow T$, then $M\left(T\left\llcorner B\left(X_{n-k}, \delta\right)\right) \leq \liminf _{j \rightarrow \infty} M\left(T_{j}\left\llcorner B\left(X_{n-k}, \delta\right)\right)\right.\right.$ and

$$
\operatorname{dist}\left(\operatorname{spt}(T), X_{n-k}\right) \geq \limsup _{j \rightarrow \infty} \operatorname{dist}\left(\operatorname{spt}\left(T_{j}\right), X_{n-k}\right) .
$$


Therefore,

$$
\ln \frac{\delta}{\operatorname{dist}\left(\operatorname{spt}(T), X_{n-k}\right)} \leq \liminf _{j \rightarrow \infty} \ln \frac{\delta}{\operatorname{dist}\left(\operatorname{spt}\left(T_{j}\right), X_{n-k}\right)}
$$

and

$$
\begin{aligned}
m_{k}^{\delta}(T) & \leq \liminf _{j \rightarrow \infty} \ln \frac{\delta}{\operatorname{dist}\left(\operatorname{spt}\left(T_{j}\right), X_{n-k}\right)} \liminf _{j \rightarrow \infty} M\left(T_{j}\left\llcorner B\left(X_{n-k}, \delta\right)\right)\right. \\
& \leq \liminf _{j \rightarrow \infty} \ln \frac{\delta}{\operatorname{dist}\left(\operatorname{spt}\left(T_{j}\right), X_{n-k}\right)} M\left(T_{j}\left\llcorner B\left(X_{n-k}, \delta\right)\right)=\liminf _{j \rightarrow \infty} m_{k}^{\delta}\left(T_{j}\right) .\right.
\end{aligned}
$$

$\therefore m_{k}^{\delta}$ is lower-semi continuous.

Case 3: $0 \leq i-k+p_{k}<n-k$ i.e. $k-p_{k} \leq i<n-p_{k}$

Remark 1. If $X$ has isolated singularities, i.e. $\operatorname{dim}(\Sigma)=0$, then case 3 will not happen.

Set

$$
G_{k}=\left\{\text { all } N-\left(i-k+p_{k}\right)-1 \text { dimensional planes in } \mathbb{R}^{N}\right\}
$$

with the standard measure $\mu$, induced from $G_{k}$ being an $\left(i-k+p_{k}\right)+1$ dimensional vector bundle over the grassmannian manifold $G\left(N, N-\left(i-k+p_{k}\right)-1\right)$ with its invariant measure.

Define $d_{T}: G_{k} \rightarrow[0,+\infty]$ by

$$
d_{T}(H)=\operatorname{dist}\left(\operatorname{spt}(T) \cap H, X_{n-k}\right) / \delta .
$$

and define

$$
u_{k}^{T}(t):=\frac{1}{t} \mu\left(d_{T}^{-1}[0, t]\right)=\frac{1}{t} \mu\left(\left\{H \in G_{k}: T\left\llcorner B\left(X_{n-k}, t \delta\right) \cap H \neq 0\right\}\right),\right.
$$

so $t u_{k}^{T}(t)$ is increasing and hence differentiable for a.e. $t \in[0,1]$.

The function $u_{k}^{T}$ gives a normalized count (without multiplicity) of the number of planes intersecting the current $T$ near the singular stratum $X_{n-k}$. It's similar to the quermassintegrale [Santalo, 13.8] of convex sets. We'll use its $\mathcal{L}^{1}$ norm to define the mass modifier in the Definition 3.0.19. Note that $\left\|u_{k}^{T}\right\|_{L^{1}([0,1])}$ may be infinite for some allowable rectifiable current $T$ having infinite order contact with $X_{n-k}$, but it will be finite for allowable subanalytic chain by Theorem 3.0.23.

To obtain the lower semicontinuity of our modified mass (defined in 3.0.19), we need the following lemmas:

Lemma 3.0.17 If $\sup M\left(T_{j}\right)+M\left(\partial T_{j}\right)<\infty$ and $T_{j} \rightarrow T$, then $u_{k}^{T}(t) \leq$ $\liminf u_{k}^{T_{j}}(t)$ for all $t \in(0,1]$. 
Proof. The hypotheses imply, by [F1, 4.3.2], that for a.e. $H \in G_{k}, T_{j} \cap H \rightarrow T \cap H$. Thus,

$$
\begin{aligned}
t u_{k}^{T}(t) & =\mu\left(\left\{H \in G_{k}: M\left(T\left\llcorner B\left(X_{n-k}, t \delta\right) \cap H\right)>0\right\}\right)\right. \\
& \leq \mu\left(\left\{H \in G_{k}: \liminf _{j \rightarrow \infty} M\left(T_{j}\left\llcorner B\left(X_{n-k}, t \delta\right) \cap H\right)>0\right\}\right)\right. \\
& =\int \chi_{\left\{H \in G_{k}: \liminf M\left(T_{j}\left\llcorner B\left(X_{n-k}, t \delta\right) \cap H\right)>0\right\}\right.} d \mu \\
& \leq \int \liminf _{j \rightarrow \infty} \chi_{\left\{H \in G_{k}: M\left(T_{j}\left\llcorner B\left(X_{n-k}, t \delta\right) \cap H\right)>0\right\}\right.} d \mu \\
& \leq \liminf _{j \rightarrow \infty} \int \chi_{\left\{H \in G_{k}: M\left(T_{j}\left\llcorner B\left(X_{n-k}, t \delta\right) \cap H\right)>0\right\}\right.} d \mu \text { (by Fatou's lemma) } \\
& =\liminf _{j \rightarrow \infty} \mu\left(\left\{H \in G_{k}: M\left(T_{j}\left\llcorner B\left(X_{n-k}, t \delta\right) \cap H\right)>0\right\}\right)\right. \\
& =t \liminf _{j \rightarrow \infty} u_{k}^{T_{j}}(t) .
\end{aligned}
$$

Lemma 3.0.18 For any $T \in \mathcal{R}_{i}(X)$, if $u_{k}^{T}(t) \in L^{1}([0,1])$, then

(1) $\mu\left(d_{T}^{-1}(0)\right)=0$;

(2) $\operatorname{dim}\left(\operatorname{spt}(T) \cap X_{n-k}\right) \leq i-k+p_{k}$;

(3) $\left\|u_{k}^{T}(t)\right\|_{L^{1}([0,1])}=\int_{d_{T}^{-1}([0,1])} \ln \left(\frac{1}{d_{T}}\right) d \mu$

Proof. Since $t u_{k}^{T}(t)=\mu\left(d_{T}^{-1}([0, t])\right)$ is increasing in $t$ and $u_{k}^{T}(t) \in L^{1}([0,1])$, we have

$$
\mu\left(d_{T}^{-1}(0)\right)=\mu\left(\left\{H: H \cap \operatorname{spt}(T) \cap X_{n-k} \neq \emptyset\right\}\right)=0 .
$$

This implies that $\operatorname{spt}(T) \cap X_{n-k}$ is a set of $\left(i-k+p_{k}\right)+1$ dimensional integral geometric Favard measure zero (see [F2] or [Santalo, III. 14.7.1] for details). By [F2, Theorem 9], $\operatorname{dim}\left(\operatorname{spt}(T) \cap X_{n-k}\right) \leq i-k+p_{k}$.

As for (3), a classical application of Fubini's theorem is the formula

$$
\int_{E} f(x) d x=\int_{0}^{\infty} m\{x \mid f(x)>t\} d t
$$

where $f$ is a nonnegative measurable function on a $\mu$ measurable set $E$.

Since $d_{T}^{-1}(0)$ has $\mu$ measure 0 , we can apply the above formula to the function $\ln \left(\frac{1}{d_{T}}\right): d_{T}^{-1}([0,1]) \rightarrow[0, \infty)$ as follows:

$$
\begin{aligned}
\int_{d_{T}^{-1}([0,1])} \ln \left(\frac{1}{d_{T}}\right) d \mu & =\int_{0}^{\infty} \mu\left(\left\{H \mid \ln \left(\frac{1}{d_{T}(H)}\right)>t\right\}\right) d t \\
& =\int_{0}^{\infty} \mu\left(\left\{H \mid 0<d_{T}(H)<e^{-t}\right\}\right) d t \\
& =\int_{0}^{1} \frac{\mu\left(\left\{H \mid 0<d_{T}(H)<s\right\}\right)}{s} d s=\int_{0}^{1} u_{k}^{T}(t) d t
\end{aligned}
$$

All our above discussion leads to the following: 
Definition 3.0.19 For fixed $\delta>0$ and any $T \in \mathcal{R}_{i}(X)$, we define the $k$-mass modifier of $T$ to be

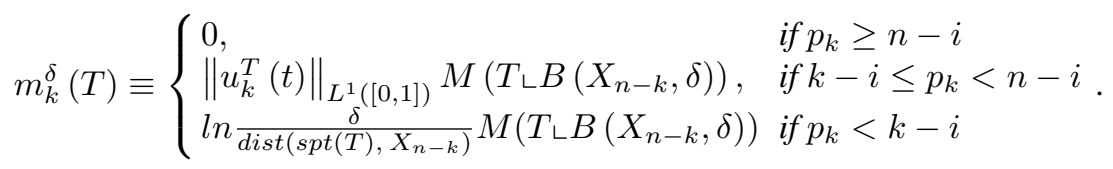

A new modified mass on $\mathcal{R}_{i}(X)$ is given by $\tilde{M}(T)=\tilde{M}^{\delta}(T) \equiv \sum_{k=2}^{n} m_{k}^{\delta}(T)+$ $M(T)$ for any $T \in \mathcal{R}_{i}(X)$. Also, we set

$$
\mathcal{I}_{i}(X)=\left\{T \in \mathcal{R}_{i}(X): \tilde{M}(T)+\tilde{M}(\partial T)<+\infty\right\}
$$

to be the set of all rectifiable currents with finite modified mass and finite modified boundary mass.

Proposition 3.0.20 If $\tilde{M}^{\delta_{0}}(T)<+\infty$ for some $\delta_{0}>0$, then $\lim _{\delta \rightarrow 0+} \tilde{M}^{\delta}(T)=$ $M(T)$.

Proof. For each $k: k-i \leq p_{k}<n-i$, whenever $\delta<\delta_{0}$,

$$
\begin{aligned}
m_{k}^{\delta}(T) & =\left\|u_{k}^{T, \delta}(t)\right\|_{L^{1}([0,1])} M\left(T\left\llcorner B\left(X_{n-k}, \delta\right)\right)\right. \\
& =\left\|\frac{\delta}{\delta_{0}} u_{k}^{T, \delta_{0}}\left(\frac{t \delta}{\delta_{0}}\right)\right\|_{L^{1}([0,1])} M\left(T\left\llcorner B\left(X_{n-k}, \delta\right)\right)\right. \\
& \leq\left\|u_{k}^{T, \delta_{0}}(t)\right\|_{L^{1}\left(\left[0, \frac{\delta}{\delta_{0}}\right]\right)} M\left(T\left\llcorner B\left(X_{n-k}, \delta_{0}\right)\right)\right. \\
& \rightarrow 0, \text { as } \delta \rightarrow 0+.
\end{aligned}
$$

Similarly, if $p_{k}<k-i$, we also have $m_{k}^{\delta}(T) \rightarrow 0$ as $\delta \rightarrow 0$. Thus, $\lim _{\delta \rightarrow 0+} \tilde{M}^{\delta}(T)=$ $M(T)$.

Proposition 3.0.21 $\tilde{M}$ mass is lower semi-continuous. i.e. if $T_{j} \rightarrow T$, then $\tilde{M}(T) \leq \liminf \tilde{M}\left(T_{j}\right)$.

Proof. For $k-i \leq p_{k}<n-i$, if $T_{j} \rightarrow T$, then by the Lemma 3.0.17, $u_{k}^{T}(t) \leq$ $\liminf u_{k}^{T_{j}}(t)$. Therefore, by the Fatou's lemma,

$$
\begin{aligned}
m_{k}^{\delta}(T) & =\left\|u_{k}^{T}(t)\right\|_{L^{1}([0,1])} M\left(T\left\llcorner B\left(X_{n-k}, \delta\right)\right)\right. \\
& \leq\left\|\liminf _{j \rightarrow \infty} u_{k}^{T_{j}}(t)\right\|_{L^{1}([0,1])} M\left(T\left\llcorner B\left(X_{n-k}, \delta\right)\right)\right. \\
& \leq \liminf _{j \rightarrow \infty}\left\|u_{k}^{T_{j}}(t)\right\|_{L^{1}([0,1])} \liminf _{j \rightarrow \infty} M\left(T_{j}\left\llcorner B\left(X_{n-k}, \delta\right)\right)\right. \\
& \leq \liminf _{j \rightarrow \infty}\left[\left\|u_{k}^{T_{j}}(t)\right\|_{L^{1}([0,1])} M\left(T_{j}\left\llcorner B\left(X_{n-k}, \delta\right)\right)\right]\right. \\
& =\liminf _{j \rightarrow \infty} m_{k}^{\delta}\left(T_{j}\right) .
\end{aligned}
$$

This, along with Lemma 3.0.16 and the lower semi-continuity of the usual mass, implies the lower semi-continuity of $\tilde{M}$. 
The following proposition says that a rectifiable current with finite modified mass and finite modified boundary mass automatically satisfies the perversity condition:

Proposition 3.0.22 $\mathcal{I}_{i}(X) \subset \mathcal{P}_{i}(X)$

Proof. For any $T \in \mathcal{I}_{i}(X)$, by the Lemma 3.0.15 and the Lemma 3.0.18, we have $\operatorname{dim}\left(\operatorname{spt}(T) \cap X_{n-k}\right) \leq i-k+p_{k}$ and $\operatorname{dim}\left(\operatorname{spt}(\partial T) \cap X_{n-k}\right) \leq i-k+p_{k}-1$ for each $k$. Thus, $T \in \mathcal{P}_{i}(X)$.

Theorem 3.0.23 $\mathcal{S}_{i}(X) \subset \mathcal{I}_{i}(X) \subset \mathcal{P}_{i}(X)$

Proof. It is sufficient to show $\mathcal{S}_{i}(X) \subset \mathcal{I}_{i}(X)$.

For any subanalytic chain $T \in \mathcal{S}_{i}(X)$ and $k$ with $k-i \leq p_{k}<n-i$, the graph of the function $d_{T}$ defined in (3) is a subanalytic set because both $\operatorname{spt}(T)$ and $X_{n-k}$ are subanalytic. By the Lojasiewicz's inequality (see Proposition 1.3.1), there exists a constant $C>0$ and $N>0$ such that $C d_{T}(H) \geq \operatorname{dist}_{G_{k}}\left(H, d_{T}^{-1}(0)\right)^{N}$. Also, $T \in \mathcal{P}_{i}(X)$ implies $\mu\left(d_{T}^{-1}(0)\right)=0$. Thus,

$u_{k}^{T}(t)=\frac{1}{t} \mu\left(d_{T}^{-1}[0, t]\right) \leq \frac{1}{t} \mu\left(\left\{H: \operatorname{dist}_{G_{k}}\left(H, d_{T}^{-1}(0)\right) \leq(C t)^{\frac{1}{N}}\right\}\right) \leq C_{1} t^{\beta-1}$

for some $\beta>0$. Therefore, $u_{k}^{T}(t) \in L^{1}[0,1]$ and hence $m_{k}^{\delta}(T)<+\infty$ for each $k$ with $k-i \leq p_{k}<n-i$. Similar arguments yield $m_{k}^{\delta}(T)<+\infty$ for all other $k$ 's. This shows $\tilde{M}(T)<+\infty$. Also, one has $\tilde{M}(\partial T)<+\infty$ because $\partial T \in \mathcal{S}_{i-1}$. Thus we have $T \in \mathcal{I}_{i}$.

Theorem 3.0.24 (Compactness theorem) Any sequence $\left\{T_{j}\right\}$ in $\mathcal{I}_{i}$ with

$$
\liminf \tilde{M}\left(T_{j}\right)+\tilde{M}\left(\partial T_{j}\right)<+\infty,
$$

contains a subsequence $\left\{T_{j_{k}}\right\}$ weakly convergent to some $T \in \mathcal{I}_{i}$.

Proof. Since lim inf $M\left(T_{j}\right)+M\left(\partial T_{j}\right)<+\infty$, by the usual compactness theorem of integer multiplicity rectifiable currents (c.f. [Simon, Theorem 27.3]), there exists a subsequence $\left\{T_{j_{k}}\right\}$ of $\left\{T_{j}\right\}$ such that $T_{j_{k}} \rightarrow T$ for some $T \in \mathcal{R}_{i}(X)$. By the lower semi-continuity of $\tilde{M}$, we have $\tilde{M}(T)+\tilde{M}(\partial T) \leq \liminf \tilde{M}\left(T_{j_{k}}\right)+$ $\tilde{M}\left(\partial T_{j_{k}}\right)<+\infty$, and hence $T \in \mathcal{I}_{i}(X)$.

A direct corollary of the previous two theorems is:

Corollary 3.0.25 Any sequence of subanalytic chains $\left\{T_{j}\right\} \subset \mathcal{S}_{i}$ with

$$
\lim \inf \tilde{M}\left(T_{j}\right)+\tilde{M}\left(\partial T_{j}\right)<+\infty
$$

contains a subsequence weakly convergent to some rectifiable current $T \in \mathcal{I}_{i}$. 


\section{$4 \tilde{M}$-mass minimizing currents}

Definition 4.0.26 We say that $T \in \mathcal{P}_{i}(X)$ is $\tilde{M}$-mass minimizing if

$$
\tilde{M}(T) \leq \tilde{M}(S)
$$

whenever $S \in \mathcal{P}_{i}(X)$ and $\partial T=\partial S$.

The following theorem says that there is a $\tilde{M}$-mass minimizer in each intersection homology class of $I H_{i}(X)$ :

Theorem 4.0.27 (Existence theorem) 1. Suppose $E=\partial S$ for some $S \in \mathcal{I}_{i}(X)$, then there exists a rectifiable current $T \in \mathcal{I}_{i}(X)$ such that $\partial T=E$ and $\tilde{M}(T) \leq \tilde{M}(R)$ for any $R \in \mathcal{I}_{i}(X)$ with $\partial R=E$.

2. For each homology class $\alpha \in I H_{i}(X)$, there exists $T \in \alpha \cap \mathcal{I}_{i}(X)$ such that $\tilde{M}(T) \leq \tilde{M}\left(T^{\prime}\right)$ for any $T^{\prime} \in \alpha$.

Proof. (1) follows from the direct method [Morgan, 1.3] and the compactness Theorem 3.0.24.

To obtain a minimizing sequence in (2), we note, by the isomorphism Theorem 2.0.14, that each class $\alpha \in I H_{i}(X)$ contains at least one subanalytic representative $S$ and $\tilde{M}(S)<+\infty$, by Theorem 3.0.23. Now, the compactness Theorem 3.0.24 ensures the existence of an $\tilde{M}$-mass minimizing in the nonempty subset $\alpha \cap \mathcal{I}_{i}(X)$.

Now, let's consider the regularity of $\tilde{M}$-Mass Minimizing currents.

For any $T \in \mathcal{I}_{i}(X)$, let $a \in \operatorname{spt}(T) \backslash(\operatorname{spt}(\partial T) \cup \Sigma)$. Recall that $a$ is a regular point of $\operatorname{spt}(T)$ if there exists an open neighborhood $U$ of $a$ in $X \backslash \Sigma$ such that $U \cap \operatorname{spt} T$ is an open $i$-manifold of class $C^{1}$.

Definition 4.0.28 Let $\omega(t)$ be defined for $0<t \leq \delta$, with $\lim _{t \rightarrow 0} \omega(t)=0$. Let $\Psi$ be a positive integrand and let $T$ be a rectifiable current with compact support in $\Omega$, an open subset of some Euclidean space $R^{N}$. Recall that $T$ is $(\Psi, \omega, \delta)$-minimal if

$$
\Psi[T\llcorner K] \leq \Psi[T\llcorner K+X]+\omega(r) M(T\llcorner K+X)
$$

for all rectifiable $X$ with compact support in $K \subset \Omega$ and with

$$
\partial X=0, \operatorname{diam}(\operatorname{spt} X) \leq r \leq \delta .
$$

Remark 4.0.29. By an argument similar to [Hardt2], the above inequality can be replaced by $\Psi[T\llcorner K] \leq \Psi[T\llcorner K+X]+\omega(r) C$ for some constant $C>0$.

Let's first recall a lemma of Almgren in [Bomb]:

Lemma 4.0.30 Let $\Psi$ be $\lambda$-elliptic and of class $C^{2}$, and let $T$ be a $(\Psi, \omega, \delta)-$ minimal current for some $\lambda<\infty, \delta>0$ and some $\omega(t)$ with

$$
\int_{0}^{\delta} \frac{\omega(t)^{1 / 2}}{t} d t<\infty .
$$

Then regular points are dense in $\operatorname{spt}(T) \backslash \operatorname{spt}(\partial T)$. 
Such current $T$ is often called an almost minimizing current. Note that this notion is very general. An oriented compact $C^{2}$ submanifold $T$ is automatically locally almost minimizing where $(\Psi, \omega, \delta)$ depend on $T$ and the distance to $\operatorname{spt}(\partial T)$.

Proposition 4.0.31 A $\tilde{M}$-mass minimizing current $T$ locally is an almost minimizing current (with $(\Psi, \omega, \delta)$ depending on $T$ and distance to spt $(\partial T) \cup \Sigma$ ).

Proof. For any $a \in \operatorname{spt} T \backslash(\operatorname{spt}(\partial T) \cup \Sigma)$, let $U_{a}$ be a very small open neighborhood of $a$ of radius $r_{a}<\frac{1}{2} \operatorname{dist}(a, \Sigma)$. Since $T$ is a $\tilde{M}$-mass minimizing current, for any i-current $S \in \mathcal{R}_{i}\left(U_{a}\right)$ with $\partial S=0$ and $\operatorname{diam}(\operatorname{spt} S) \leq r \leq r_{a}$, we have

$$
\tilde{M}(T) \leq \tilde{M}(T+S) .
$$

For each $k=2, \cdots, n$, let $\Phi_{k}$ be the nonnegative integrand whose total integral over a rectifiable current $R$ is

$$
\Phi_{k}(R) \equiv h_{k} M\left(R\left\llcorner B\left(X_{n-k}, \delta\right)\right)\right.
$$

where

$$
h_{k}=\left\{\begin{array}{ll}
\left\|u_{k}^{T}\right\|_{L^{1}[0,1]}, & \text { if } \operatorname{dist}\left(a, X_{n-k}\right) \leq \delta \text { and } k-i \leq p_{k}<n-i \\
\ln \frac{\delta}{\operatorname{dist}\left(\operatorname{spt}(T), X_{n-k}\right)}, & \text { if } \operatorname{dist}\left(a, X_{n-k}\right) \leq \delta \text { and } p_{k} \geq n-i \\
0, & \text { otherwise }
\end{array} .\right.
$$

Lemma 4.0.32 For each $k=2, \cdots, n$ and $r$ small enough,

$$
\begin{aligned}
& {\left[\Phi_{k}(T)-\Phi_{k}(T+S)\right]-\left[m_{k}^{\delta}(T)-m_{k}^{\delta}(T+S)\right] } \\
\leq & C_{k} \frac{r}{\operatorname{dist}\left(a, X_{n-k}\right)} M\left((T+S)\left\llcorner B\left(X_{n-k}, \delta\right)\right)\right.
\end{aligned}
$$

for some positive constant $C_{k}$ independent of $T, S$ and $a$.

Proof. If $\operatorname{dist}\left(a, X_{n-k}\right)>\delta$, we may choose $U_{a}$ small enough such that $\operatorname{dist}\left(U_{a}, X_{n-k}\right)>\delta$, then $m_{k}^{\delta}(T)=m_{k}^{\delta}(T+S)$.Thus,

$$
\left[\Phi_{k}(T)-\Phi_{k}(T+S)\right]-\left[m_{k}^{\delta}(T)-m_{k}^{\delta}(T+S)\right]=[0-0]-0=0 .
$$

Now, we assume $\operatorname{dist}\left(a, X_{n-k}\right) \leq \delta$. In this case, $\Phi_{k}(T)=m_{k}^{\delta}(T)$, so it is sufficient to show

$$
m_{k}^{\delta}(T+S)-\Phi_{k}(T+S) \leq C_{k} \frac{r}{\operatorname{dist}\left(a, X_{n-k}\right)} M\left((T+S)\left\llcorner B\left(X_{n-k}, \delta\right)\right)\right.
$$

1. If $p_{k} \geq n-i$, then $m_{k}^{\delta}(T+S)-\Phi_{k}(T+S)=0-0=0$. 
2. If $k-i \leq p_{k}<n-i$, then for any $t \in(0,1]$, we consider

$$
\begin{aligned}
& t\left(u_{k}^{T+S}(t)-u_{k}^{T}(t)\right) \\
= & \mu\left\{H \in G_{k}: \operatorname{spt}(T+S) \cap B\left(X_{n-k}, t \delta\right) \cap H \neq \emptyset\right\} \\
& -\mu\left\{H \in G_{k}: \operatorname{spt}(T) \cap B\left(X_{n-k}, t \delta\right) \cap H \neq \emptyset\right\} \\
\leq & \mu\left\{H \in G_{k}: \operatorname{spt}(T+S) \cap B\left(X_{n-k}, t \delta\right) \cap H \neq \emptyset\right. \\
& \text { and } \left.\operatorname{spt}(T) \cap B\left(X_{n-k}, t \delta\right) \cap H=\emptyset\right\} \\
= & \mu\left\{H \in G_{k}: \operatorname{spt}(S) \cap B\left(X_{n-k}, t \delta\right) \cap H \neq \emptyset\right. \\
& \text { and } \left.\operatorname{spt}(T) \cap B\left(X_{n-k}, t \delta\right) \cap H=\emptyset\right\} \\
\leq & \mu\left\{H \in G_{k}: U_{r} \cap B\left(X_{n-k}, t \delta\right) \cap H \neq \emptyset\right\} \\
\leq & \begin{cases}0, & \text { if } t \delta \leq \operatorname{dist}\left(U_{a}, X_{n-k}\right) \\
C_{k} r^{\left(i-k+p_{k}\right)+1} & \text { if } t \delta \geq \operatorname{dist}\left(U_{a}, X_{n-k}\right)\end{cases}
\end{aligned}
$$

where $U_{r} \subset U_{r_{a}}$ is an Euclidean ball of radius $r$ that contains $S$ and $C_{k}$ is a constant depends only on $N$ and $\left(i-k+p_{k}\right)+1$, the dimension of the moving planes. Note the last inequality follows from the formula ([Santalo, 13.46]) for the quermassintegrale of a ball. Thus, we have

$$
\begin{aligned}
& \left\|u_{k}^{T+S}\right\|_{L^{1}[0,1]}-\left\|u_{k}^{T}\right\|_{L^{1}[0,1]} \\
= & \int_{0}^{1} u_{k}^{T+S}(t)-u_{k}^{T}(t) d t \\
\leq & \int_{\operatorname{dist}\left(U_{a}, X_{n-k}\right) / \delta}^{1} \frac{C_{k}}{t} r^{\left(i-k+p_{k}\right)+1} d t \\
\leq & C_{k} r^{\left(i-k+p_{k}\right)+1} \ln \frac{\delta}{\operatorname{dist}\left(U_{a}, X_{n-k}\right)} \\
\leq & C_{k} r \frac{2 \delta}{\operatorname{dist}\left(a, X_{n-k}\right)}
\end{aligned}
$$

Hence,

$$
\begin{aligned}
& m_{k}^{\delta}(T+S)-\Phi_{k}(T+S) \\
= & {\left[\left\|u_{k}^{T+S}\right\|_{L^{1}[0,1]}-\left\|u_{k}^{T}\right\|_{L^{1}[0,1]}\right] M\left((T+S)\left\llcorner B\left(X_{n-k}, \delta\right)\right)\right.} \\
\leq & 2 \delta C_{k} \frac{r}{\operatorname{dist}\left(a, X_{n-k}\right)} M\left((T+S)\left\llcorner B\left(X_{n-k}, \delta\right)\right) .\right.
\end{aligned}
$$


3. If $p_{k}<k-i$, then when $r$ small enough,

$$
\begin{aligned}
& \ln \frac{\delta}{\operatorname{dist}\left(\operatorname{spt}(T+S), X_{n-k}\right)}-\ln \frac{\delta}{\operatorname{dist}\left(\operatorname{spt}(T), X_{n-k}\right)} \\
= & \begin{cases}0, & \text { if } \operatorname{dist}\left(a, X_{n-k}\right)>\operatorname{dist}\left(\operatorname{spt}(T), X_{n-k}\right) \\
\ln \frac{\operatorname{dist}\left(\operatorname{spt}(T), X_{n-k}\right)}{\operatorname{dist}\left(\operatorname{spt}(T+S), X_{n-k}\right)} & \text { if } \operatorname{dist}\left(a, X_{n-k}\right)=\operatorname{dist}\left(\operatorname{spt}(T), X_{n-k}\right)\end{cases} \\
\leq & \begin{cases}0, & \text { if } \operatorname{dist}\left(a, X_{n-k}\right)>\operatorname{dist}\left(\operatorname{spt}(T), X_{n-k}\right) \\
\ln \frac{\operatorname{dist}\left(\operatorname{spt}(T), X_{n-k}\right)}{\operatorname{dist}\left(\operatorname{spt}(T), X_{n-k}\right)-r} & \text { if } \operatorname{dist}\left(a, X_{n-k}\right)=\operatorname{dist}\left(\operatorname{spt}(T), X_{n-k}\right)\end{cases} \\
\leq & \frac{2 r}{\operatorname{dist}\left(a, X_{n-k}\right)} .
\end{aligned}
$$

Therefore, by the definition of $h_{k}$ in (4),

$$
\begin{aligned}
& m_{k}^{\delta}(T+S)-\Phi_{k}(T+S) \\
= & \left(\ln \frac{\delta}{\operatorname{dist}\left(\operatorname{spt}(T+S), X_{n-k}\right)}-h_{k}\right) M\left((T+S)\left\llcorner B\left(X_{n-k}, \delta\right)\right)\right. \\
\leq & \frac{2 r}{\operatorname{dist}\left(a, X_{n-k}\right)} M\left((T+S)\left\llcorner B\left(X_{n-k}, \delta\right)\right) .\right.
\end{aligned}
$$

Now, consider the positive integrand $\Psi=\sum_{k=2}^{n} \Phi_{k}+\Phi$, where $\Phi$ is the area integrand. Since $T$ is $\tilde{M}$-mass minimizer, by the above Lemma 4.0.32,

$$
\begin{aligned}
& \Psi(T)-\Psi(T+S) \\
\leq & \Psi(T)-\Psi(T+S)+\tilde{M}(T+S)-\tilde{M}(T) \\
= & \sum_{k=2}^{n}\left[\Phi_{k}(T)-\Phi_{k}(T+S)\right]-\left[m_{k}^{\delta}(T)-m_{k}^{\delta}(T+S)\right] \\
\leq & \sum_{k=2}^{n} C_{k} \frac{r}{\operatorname{dist}\left(a, X_{n-k}\right)} M\left((T+S)\left\llcorner B\left(X_{n-k}, \delta\right)\right)\right. \\
\leq & \sum_{k=2}^{n} C_{k} \frac{r}{\operatorname{dist}(a, \Sigma)} M((T+S)\llcorner B(\Sigma, \delta)) \\
= & \frac{C}{\operatorname{dist}(a, \Sigma)} r M(T\llcorner B(\Sigma, \delta)+S)
\end{aligned}
$$

where $C=\sum_{k=2}^{n} C_{k}$ is a constant. Thus,

$$
\Psi\left(T\left\llcorner U_{a}\right) \leq \Psi\left(T\left\llcorner U_{a}+S\right)+\frac{C}{\operatorname{dist}(a, \Sigma)} r M(T\llcorner B(\Sigma, \delta)+S) .\right.\right.
$$

This implies $T$ is $\left(\Psi, \frac{C}{\operatorname{dist}(a, \Sigma)} r, r_{a}\right)$-minimal at $a$.

Corollary 4.0.33 (Regularity Theorem) Let $T$ be a $\tilde{M}$-mass minimizing current of $X$, then the regular points are dense in $\operatorname{spt}(T) \backslash(\operatorname{spt}(\partial T) \cup \Sigma)$. 


\section{Some simple examples}

Example 1 Consider the stratified space $\mathbb{R}^{2} \supset\{0\}$ with a single singular point $\{0\}$. Suppose $p, q \in \mathbb{R}^{2} \backslash\{0\}$ such that 0 is on the line segment $\overline{p q}$. Then under the usual mass of $\mathbb{R}^{2}$, the minimal path from $p$ to $q$ is the line segment $\overline{p q}$ which passes through the singular point $\{0\}$ and hence does not satisfy the allowability condition. Given any small number $\delta>0$, one can easily check that the minimum path from $p$ to $q$ under the modified mass is the minimum path from $p$ to $q$ in the domain $\mathbb{R}^{2} \backslash B_{\delta}(0)$. A typical example looks like the following graph:

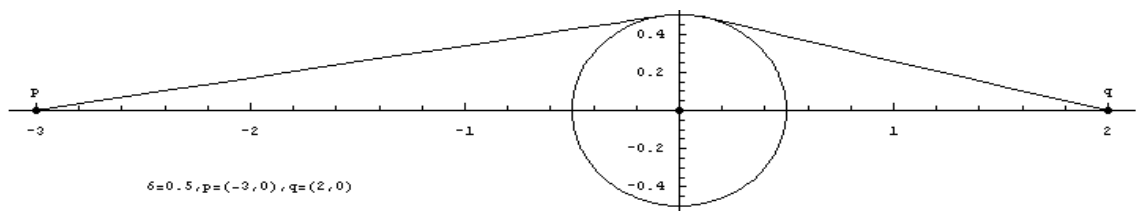

Fig. 1. $p=(-3,0), q=(2,0)$ and $\delta=0.5$

Example 2 Consider Whitney umbrella $W: x^{2}-z y^{2}=0$ in $\mathbb{C}^{3}$ with its Whitney stratification: $W \supseteq W_{2} \supseteq W_{0}$, where $W_{2}$ is the complex $z$-axis defined by $x=$ $y=0$ and $W_{4}$ is the point $\{(0,0,0)\}$.

Note that for any nonzero complex number $z_{0}$, there are two associated real 2 dimensional planes $x^{2}=z_{0} y^{2}$. These two planes coincide at 0 to the plane $x=z=0$.

Now, let's consider some simple variational problems on this famous stratified space.

Case 1: $i=1$. Since $W \backslash W_{2}$ is path connected, any two points $p, q \in W \backslash W_{2}$ can be joined by some path inside $W \backslash W_{2}$. Let $\gamma_{p q} \subset W$ be a length minimizer from $p$ to $q$ under the usual mass. If $\gamma_{p q}$ does not intersect the singular part $W_{2}$, i.e. $\gamma_{p q}$ is $(\bar{p}, 1)$-allowable, then when $\delta$ is small enough, $\gamma_{p q}$ will also be a minimizer from $p$ to $q$ under the modified mass. If $\gamma_{p q}$ intersects $W_{2}$, then there exists a modified

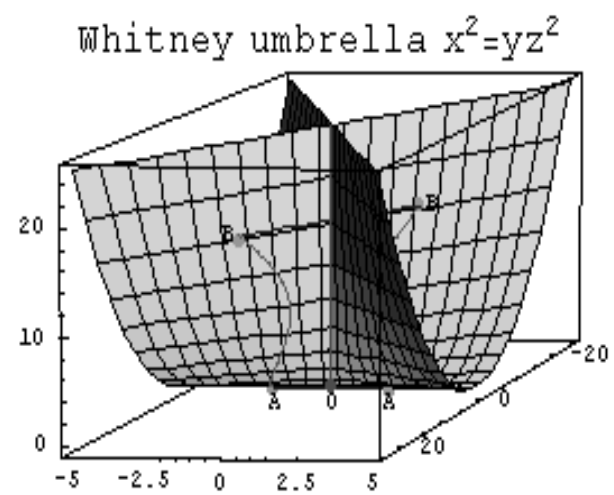


mass minimizer $\bar{\gamma}_{p q}$ from $p$ to $q$ which does not intersect the singular part $W_{2}$. The shape of $\bar{\gamma}_{p q}$ looks like the graph in the previous example.

Case 2: $i=2$. In this case, the crucial number $i-j+p_{j}$ in the perversity condition is given by

$$
i-j+p_{j}=\left\{\begin{array}{l}
0, \quad j=2 \text { and } p_{2}=0 \\
-2, j=4 \text { and } p_{4}=0 \\
-1, j=4 \text { and } p_{4}=1 \\
0, \quad j=4 \text { and } p_{4}=2
\end{array}\right. \text {. }
$$

This means that $(\bar{p}, 2)$-allowable chains are allowed to intersect $W_{2}$ with 0 dimensional set but are not allowed to intersect $W_{4}$ unless $p_{4}=2$.

Now, let's consider two $(\bar{p}, 1)$-allowable circles in $W$ :

$A=\left\{\left(0, e^{i \theta}, 0\right): \theta \in[0,2 \pi]\right\}$, the unit circle on the plane $x=z=0$ (i.e. on the complex $y$-axis);

$B=\left\{\left(z_{0} e^{i \theta}, e^{i \theta}, z_{0}^{2}\right): \theta \in[0,2 \pi]\right\}$, a circle around $\left(0,0, z_{0}^{2}\right)$ on one of the two planes $x^{2}=z_{0}^{2} y^{2}$ associated to some $z_{0} \neq 0$.

Under the usual mass, the minimal surface having boundary $A-B$ will be either a catenoid or union of two disjoint unit disks, depending on the location of $z_{0}$. In the later situation, the centers of these two unit disks are $(0,0,0) \in W_{4}$ and $\left(0,0, z_{0}^{2}\right) \in W_{2} \backslash W_{4}$, lying on the singular sets. Therefore, when $p_{4}=0$ or 1 , the union of two unit disks does not satisfy the desired perversity conditions.

Under the modified mass, the corresponding minimal surface with boundary $A-B$ will still be either a catenoid or union of two disjoint slightly curved disks. However, in the later situation, the centers of the disks are no longer touching $W_{4}$, they all lie on $W_{2} \backslash W_{4}$ now. This makes the minimal surface to be $(\bar{p}, 2)$-allowable as desired. Their graphs are shown in the following diagram:

\begin{tabular}{|l|l|l|}
\hline$i=2$ & $p_{\mathrm{d}}=0$ or 1 & $p_{\mathrm{d}}=2$ \\
\hline \multirow{4}{*}{ minimizer from $A$ to $B$} & or & \\
& & \\
\hline
\end{tabular}

Example 3 Consider the stratified space $X \supseteq X_{2} \supseteq X_{0}$ with $X=\mathbb{R}^{4}=$ $\{(x, y, z, t)\}, X_{2}=\{(x, y, z, t) \mid x=y=0\}$ and $X_{0}=\{(0,0,0,0)\}$. Let

$$
T=\left\{\left(2+\cos \theta\left(2+\frac{\cos \alpha}{2}\right), \sin \theta\left(2+\frac{\cos \alpha}{2}\right), \frac{\sin \alpha}{2}, 0\right): \theta, \alpha \in[0,2 \pi]\right\}
$$

be a torus inside $X$. Note that $T$ is $(\bar{p}, 2)$-allowable for any perversity $\bar{p}$. By using the convex hull property of mass minimizers, one easily see that the 3 -dimensional volume minimizer having boundary $T$ (under the usual mass) is simply the solid torus $S$. Now, $S \cap X_{2}=\left\{(0,0, z, 0): z \in\left[-\frac{1}{2}, \frac{1}{2}\right]\right\}$ has dimension 1 and passes 
through $X_{0}$. Thus, when $p_{4}=1$ or $2, S$ is $(\bar{p}, 3)$-allowable. However, when $p_{4}=0$, $S$ becomes not allowable. In this case, one can use our modified mass to get a modified mass minimizer $S^{\prime}$ in $X$, having boundary $T$ and is $(\bar{p}, 3)$-allowable. In other words, this modified mass minimizer doesn't intersect the singular point $\{(0,0,0,0)\}$.

\section{Part of the torus T}

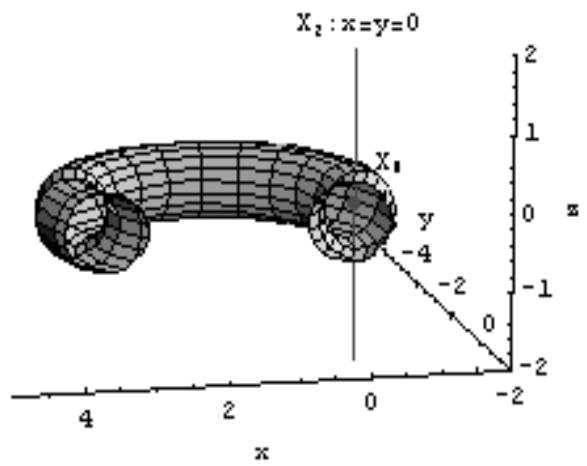

\section{References}

[Bomb] E. Bombieri: Regularity theory for almost Minimal currents, p. 99-130.

[Borel] A. Borel et al.: Intersection cohomology, Progress in mathematics 50. Birkhäuser Boston, Basel 1984.

[F1] H. Federer: Geometric Measure Theory. Springer-Verlag, New York, 1969.

[F2] H. Federer: Dimension and measure. Trans. Amer. Math. Soc. 62 (1947), 536547.

[GM1] M. Goresky, R. MacPherson: Intersection homology theory. Topology 19 (1980), 135-162.

[GM2] M. Goresky, R. MacPherson: Intersection homology theory II. Inv. Math. 72 (1983), 77-130.

[Hardt1] R. Hardt, L. Simon: Seminar on Geometric Measure Theory. Birkhäuser, Boston, 1986.

[Hardt2] R. Hardt, D. Kinderlehrer: Some regularity results in ferromagnetism. Comm. Partial Differential Equations 25 (2000), 1235-1258.

[Hardt3] R. Hardt: Topological properties of subanalytic sets. Trans. Amer. Math. Soc. 211 (1975), 57-70.

[Hardt4] R. Hardt: Triangulation of subanalytic sets and proper light subanalytic maps. Invent. Math. 38 (1976/77), 207-217.

[Hironaka] H. Hironaka: Introduction to Real-Analytic sets and real-analytic maps. Istituto Matematico “L.Tonelli” Dell' Universita'DI Pisa, 1973.

[Kirwan] F. Kirwan: An introduction to intersection homology theory. Longman Scientific \& Technical, 1988.

[Li] M. Li; Currents on spaces with cone-like singularities. Ph.D thesis, Rice University, 1995.

[Morgan] F. Morgan: Geometric Measure theory, a beginner's guide. Academic Press, 1995. 
[Santalo] L.A. Santalo: Integral Geometry and Geometric Probability. Encyclopedia of mathematics and its applications, Volume I 1976.

[Simon] L. Simon: Lectures on geometric measure theory. Proc. Centre Math. Anal. Australian National University 3 (1983) 